\title{
Avaliação da Qualidade de Vida e das Variáveis Clínicas no Desempenho Físico dos Pacientes com Insuficiência Cardíaca Crônica
}

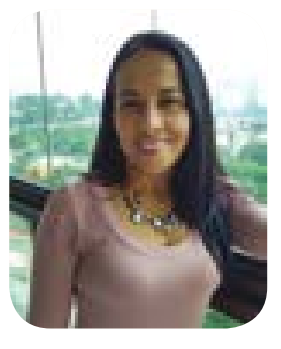

RESUMO

INTRODUÇÃO: a qualidade de vida e o desempenho físico dos pacientes com insuficiência cardíaca estão relacionados com o prognóstico da doença, limitando em realizar as atividades de vida diária necessárias a uma vida independente. OBJETIVO: comparar o desempenho físico (DF) e a qualidade de vida (QVD) dos pacientes clínicos com Insuficiência Cardíaca Crônica (ICC) entre as Classes Funcionais (CF) II e III. METODOLOGIA: trata-se de um estudo transversal e descritivo, onde foram analisados 70 pacientes portadores de ICC Classe Funcional II/III ou Estágio $B$ e $C$, sendo aplicado o questionário de qualidade de vida (MINNESOTA) e verificadas as variáveis clínicas através do teste de caminhada para a avaliação do desempenho físico. RESULTADOS: foram avaliados 70 pacientes, sendo predominante 0 sexo feminino, $39(55,7 \%)$, com a média total de peso e idade, respectivamente, de $65,16 \mathrm{~kg}( \pm 14,31)$ e 54 ( \pm 15$)$ anos. Quanto ao perfil, $42(60,0 \%)$ dos indivíduos clínicos pertenciam à Classe Funcional II (CF II). Dentre as principais causas da ICC entre os indivíduos entrevistados, 38 (55,9\%) possuíam Miocardiopatia Chagásica (MCC). E dos fatores de risco, a maior incidência foi de ex-tabagistas, representada por 30 $(42,9 \%)$ pacientes. Na análise da disfunção ventricular observou-se que, na CF II, 20 (55,6\%) possuíam a disfunção sistólica e 16 (44,8\%), disfunção diastólica. Ao analisar o questionário Minnesota, comparando as classes funcionais, foi observado que a capacidade física teve melhor resultado na CF II: 20,92 $( \pm 10,25)$. CONCLUSÃO: portanto, quando comparados, os indivíduos de CF II e CF III, à medida que se avança a classe funcional da IC, menor será o desempenho físico e a qualidade de vida, estando diretamente relacionada ao impacto da progressão da dispneia.
PALAVRAS-CHAVE: insuficiência cardíaca; qualidade de vida; Minessota; teste de caminhada.

\section{INTRODUÇÃO}

A Insuficiência Cardíaca (IC) é uma síndrome clínica complexa, classicamente definida como incapacidade do coração em bombear suprimentos adequados de sangue em relação ao retorno venoso e às necessidades metabólicas tissulares, ocasionando redução do débito cardíaco e elevação das pressões pulmonares e da venosa sistêmica. (SOARES, D.A. et. al., 2008; FIGUEROA, M.S. and PETERS, J.I, 2006; GUYTON, A.C. e HALL, J.E.,2008)

A IC é causada por diversas etiologias, como: doença aterosclerótica do coração, hipertensão arterial, estenose da válvula aórtica, miocardiopatia chagásica, miocardiopatia alcoólica, miocardites, doenças valvulares e cardiopatias congênitas. (BOCCHI, E.A. et. al., 2005)

Atualmente, existem duas classificações utilizadas para categorizar a Classificação Funcional (CF) nos indivíduos que sofrem de IC. Essas classificações podem ser feitas através da New York Heart Association (NYHA), que é dada de acordo com o grau de dispneia e fadiga durante o mínimo e/ou máximo esforço, sendo avaliada também no repouso. Quanto mais evidente a sintomatologia, mais grave é o paciente. $E$ a segunda, da American Heart Association (AHA) / American College of Cardiology (ACC), que é distribuída em estágios, baseados na mudança da cardiopatia estrutural e dos sintomas da IC. (SMITH, S.C. et. al.,2001)

Contudo, a progressão da dispneia na IC piora o prognóstico e a qualidade de vida, devido à redução da força e da resistência dos músculos respiratórios, provocando uma resposta limitada ao desempenho físico. (CHIAPPA, G.R. et. al., 2008; ATHANASIOS, T. et. al., 2010) 
Segundo a Organização Mundial da Saúde (OMS), a Qualidade de Vida (QV) é definida como "a percepção do indivíduo em relação à sua posição na vida, de acordo com o contexto cultural e os sistemas de valores nos quais vive e em relação a seus objetivos, expectativas, padrões e preocupações". (SANTOS, J.J.A. e cols, 2009; DANTAS,R.A.S e cols, 2003; SEILD, E.M.F e ZANNON, C.M.L.C, 2004)

Todavia, para avaliar a Qualidade de Vida em pacientes com Insuficiência Cardíaca, a literatura apresenta questionários genéricos e específicos, dos quais o Minnesota Living with Heart Failure Questionnaire (MLHFQ) é um dos mais utilizados e foi desenvolvido especificamente para IC, o que torna mais próximo à realidade desse tipo de paciente, avaliando sua condição de enfermidade. (DANTAS,R.A.S e cols, 2003; NOGUEIRA, I.D.B e cols, 2010; CARVALHO, V.O e cols, 2009; PILON, A.F, 1992)

O MLHFQ pode auxiliar os profissionais de saúde a planejarem e aplicarem estratégias de tratamento para os portadores de disfunção ventricular, proporcionando-Ihes uma melhor expectativa de vida (SANTOS, J.J.A. e cols, 2009). Outro aspecto importante sobre este instrumento é seu formato abrangente, fácil de aplicar, adequado para pacientes idosos e com validação para a população brasileira. (CARRARA, D., 2001; SACCOMANN, I.C. e cols, 2007)

O objetivo do questionário Minnesota é medir a satisfação do paciente em três dimensões: física, emocional e global, considerando as relações interpessoais com o mundo em que ele vive, interferindo em seu estado íntimo, físico e social. (CARVALHO, V.O e cols, 2009; PILON, A.F, 1992)

Entretanto, para avaliar a desempenho físico, o teste de caminhada é um método de avaliação utilizado para verificar os efeitos de intervenções terapêuticas, como na insuficiência cardíaca e reabilitação pulmonar. (BANSAL, V. et al, 2008)

O teste de caminhada (TC6') consta de uma técnica alternativa simples, de fácil execução, porém bem tolerada e precisa para a medição da capacidade de esforço submáxima em pacientes portadores de IC. (FUMAGALLI, E. e cols, 2010; LI, A.M. e cols, 2005; ENRIGHT, P.L, 2003; BERISHA, V. e cols, 2009).

Para a execução do TC6' é aferido o Índice de Esforço Percebido, através da Escala de Borg, antes e depois do teste, pois verifica subjetivamente o cansaço do paciente (FUMAGALLI, E. e cols, 2010). A distância percorrida em 6 minutos (TC6') tem sido mostrada com precisão e predição das morbimortalidades por doenças cardiopulmonares (ASSOCIATION, AH, 2007).
Entretanto, o teste é interrompido em indivíduos que apresentem dor precordial, dispneia desproporcional ao esforço, sudorese excessiva, palidez ou indisposição. (SOUSA, L.A.P, 2008)

De acordo com as repercussões funcionais e limitações vivenciadas pelos pacientes com acometimento cardíaco, a dispneia, intolerância ao esforço, a consequente limitação ao exercício, às atividades de vida diária e laborativa podem ser os fatores fisiopatológicos apresentados pela população de pacientes com falência cardíaca. (SOUSA, L.A.P, 2008)

O presente estudo foi desenvolvido para avaliar as variáveis clínicas quanto à influência da IC na qualidade de vida e no desempenho físico entre as Classes Funcionais II e III.

\section{MATERIAIS E MÉTODOS}

\section{População do estudo}

Trata-se de um estudo de campo descritivo, composto por 70 pacientes portadores de Insuficiência Cardíaca Crônica de Classe Funcional II/III ou Estágio B e C, atendidos no Ambulatório Silva e Lima do Hospital Santa Izabel, no período entre março a junho de 2010. Os pacientes foram divididos em dois grupos: grupo 1 (ICC CF II) e grupo 2 ( ICC CF III).

Os critérios para inclusão no estudo foram: os pacientes de ambos os sexos, com idade superior a 18 anos, que apresentaram ICC CF II/III ou no estágio B e C, com disfunção sistólica e/ou diastólica e clínicos confirmados com diagnóstico do médico cardiologista, além de apresentarem autonomia em realizar suas atividades de vida diária e que assinaram o Termo de Consentimento Livre e Esclarecido.

Os critérios de exclusão foram pacientes com Insuficiência Cardíaca Classes I e IV ou no estágio A e D, com limitação física de origem articular, neurológica e/ ou metabólica.

\section{MÉTODO DE AVALIAÇÃO}

\section{Avaliação das variáveis clínicas}

$\mathrm{Na}$ avaliação das Variáveis Clínicas, o paciente encontrava-se na posição sentada, por pelo menos cinco minutos. Através do método auscultatório, com esfigmomanômetro Tycos ${ }^{\circledR}$ e o estetoscópio Littmann, foi mensurada a Pressão Arterial (PA). A Frequência Cardíaca (FC) e SatO2 (\%) foram verificadas através de um oxímetro de pulso CM 50D (Contec medical system).

A Frequência Cardíaca Máxima foi calculada através da fórmula de karvonen: 220 - idade do paciente. (WILLIAM, E. D.T. et. al., 2008). 


\section{Avaliação Antropométrica}

Para medidas antropométricas de peso corporal e estatura, foi utilizada a balança Welmy (Indústria e Comércio Ltda. com $\mathrm{n}^{\circ}$ de patrimônio 007953).

O Índice de Massa Corporal (IMC), segundo a OMS (Organização Mundial de Saúde), foi classificado como: baixo peso $(<18,5)$; normal $(18,5-24,9 \mathrm{~kg} /$ $\mathrm{m} 2)$, acima do peso $(25-29,9 \mathrm{~kg} / \mathrm{m} 2)$ e obeso $(\geq 30.0$ $\mathrm{kg} / \mathrm{m} 2$ ), (WHO, 1998), e calculado de acordo com a fórmula: peso $(\mathrm{kg})$ dividido pela altura $(\mathrm{m} 2)$. (SWARTZ, A.M e cols, 2003; SWARTZ, A.M. e cols, 2009).

\section{Qualidade de Vida}

$\mathrm{Na}$ avaliação da qualidade de vida, através de entrevista, foi utilizado, em ambos os grupos, na versão brasileira, o Minnesota Living with Heart Failure Questionnaire (MLHFQ), cuja pontuação de 0 a 5 considera respostas como: nenhum, muito pouco, pouco, mais ou menos, muito e bastante, tendo o escore total de 0 a 105, resultante da somatória das 21 questões, onde um escore mais baixo corresponde a uma melhor QDV. O instrumento é aplicado usando uma escala de seis pontos, que variam de 0 (nenhum) a 5 (bastante), sendo que número 0 representa sem limitações e o número 5 sinaliza limitação máxima. (SOARES, D.A. et. al., 2008; DANTAS, R.A.S. e cols, 2003; NOGUEIRA, I.D.B. e cols, 2010; RIEGEL, B. et. al., 2002; MILLER, K. e cols, 2004).

O formato de resposta foi escolhido por ser coerente com o conceito de qualidade de vida e permitir a cada indivíduo responder cada pergunta através desta escala. As questões são divididas em três aspectos: o físico, que corresponde às questões de 1 a 7, 12 e 13; emocional, do número 17 ao 21 ; e as demais, relacionadas como dimensões gerais. (SOARES, D.A. et. al., 2008; DANTAS, R.A.S. e cols, 2003; NOGUEIRA, I.D.B. e cols, 2010; RIEGEL, B. et. al., 2002; MILLER, K. e cols, 2004).

Os sintomas físicos são percebidos principalmente através da dispneia e da fadiga, durante as atividades habituais. Os psicológicos, através da perda de autocontrole em sua vida e da sensação de ser um peso para o próximo. E no impacto global é questionado o uso de medicamentos que causam efeitos colaterais. Inclui-se também a parte social, como por exemplo o impacto da atividade no trabalho, no relacionamento, com a família ou amigos e no lazer. (SOARES, D.A. et. al., 2008; DANTAS, R.A.S. e cols, 2003; NOGUEIRA, I.D.B. e cols, 2010; RIEGEL, B. et. al., 2002; MILLER, K. e cols, 2004).

\section{Teste de Caminhada de 6 Minutos}

Para avaliar o desempenho físico foi utilizado o Teste de Caminhada de 6 minutos, com a variável
Distância Percorrida e variáveis clínicas, como: Pressão Arterial (PA), Frequência Cardíaca (FC), Frequência Cardíaca Máxima (FCM), Saturação de Oxigênio (Sato2) e Escala de BORG.

Todos os pacientes foram informados do objetivo, métodos e uso dos resultados do teste de caminhada de 6 minutos e da utilização do pedômetro, aparelho prático e portátil que serve para medir, durante a marcha, os registros da distância percorrida, o número de passos e estimar o dispêndio energético em quilocalorias. (LOPES, V.P. et. al., 2003; PINTO, C.N.C, 2008; OLIVEIRA, M. et. al., 2003; SWARTZ, A.M. e cols, 2003; SWARTZ, A.M. e cols, 2009).

Neste estudo, o pedômetro foi posicionado no lado esquerdo, a $2 \mathrm{~cm}$ acima da Espinha llíaca Antero-Superior (EIAS), sendo registrados somente os valores da distância percorrida.

O TC6' foi realizado em uma superfície plana, conforme o protocolo padronizado do ATS (American Thoracic Society), em um corredor plano de $30 \mathrm{~m}$, livre de obstáculos, sinalizado por cones (cone de trânsito amarelo e preto), onde os pacientes foram orientados a andar a maior distância possível, virando $180^{\circ}$ a cada $30 \mathrm{~m}$, dentro do limite de tempo de seis minutos. (CRAPO, R.O. e cols, 2002)

Através do protocolo preestabelecido, os pacientes foram orientados, de acordo com sua tolerância, caminhar no período de seis minutos, onde andavam livres, sem interferência física do avaliador e somente era informado o tempo a cada minuto que necessitava para finalizar o percurso. As variáveis Pressão Arterial (PA), Frequência Cardíaca (FC) e Saturação de Oxigênio $\left(\mathrm{SatO}_{2}\right)$ foram mensuradas no início e ao final do TC6'. A percepção subjetiva da escala de BORG foi somente ao final do teste.

\section{Aspectos Éticos}

Este estudo foi aprovado pelo Comitê de Ética e Pesquisa do Hospital Santa Izabel (HSI), onde não apresentou qualquer situação de constrangimento ou lesão dos envolvidos, bem como se manteve a confidencialidade e o anonimato dos envolvidos.

Todos os participantes foram convidados a participar da pesquisa e informados sobre a mesma, na qual obtiveram aceitação através da permissão e assinatura do Termo de Consentimento Livre e Esclarecido, de acordo com as recomendações da Resolução 196/96 do Conselho Nacional da Saúde do Brasil.

\section{Análise Estatística}

Criado no Excel 2003, o banco de dados foi transferido e analisado no Software SPSS, versão 14.0, 
mediante prévia correção dos dados digitados, com o objetivo de eliminar possíveis erros.

Os dados foram analisados por estatística descritiva, expressa em valores absolutos e relativos em percentual (variáveis categóricas), e média com desvio padrão quando a distribuição era normal ou mediana, com intervalo interquartil, quando a distribuição não era homogênea (variáveis contínuas), onde foi realizado com a finalidade de identificar as características gerais e específicas da amostra estudada.

Para verificar a associação entre Qualidade de Vida e a Classe Funcional, utilizou-se o teste paramétrico T - Student e para a associação entre a Classe Funcional e o Desempenho Físico foi usado o Mann-Whitney Test, considerando o nível de significância de 5\%. Os resultados obtidos foram apresentados em tabelas comparativas, formuladas no Word.

\section{RESULTADOS}

Foram analisados 70 indivíduos, com predomínio do sexo feminino 55,7\%. Quanto à Classe Funcional dos pacientes, foi observada maior presença $(60,0 \%)$ de indivíduos da Classe Funcional II do ICC.

Somente 60 pacientes possuíam os exames de ecocardiograma utilizados para a confirmação da disfunção sistólica, origem sistólica ou diastólica, sendo que a maior incidência de disfunção ventricular foi de com $(55,6 \%)$ na CF II e $(70,8 \%)$ CF III. (Tabela 1$)$.

Tabela 1 - Categorização das variáveis clínicas quanto à Classe Funcional dos pacientes do Ambulatório Silva e Lima do HSI, 2010.

\begin{tabular}{|l|l|l|l|l|l|l|l|}
\hline \multirow{3}{*}{ VARIÁVEIS } & \multicolumn{7}{|c|}{ PERFIL } \\
\cline { 2 - 8 } & CF II (n=42) & \multicolumn{2}{|c|}{ CF III (n=28) } & \multicolumn{4}{c|}{ TOTAL } \\
\cline { 2 - 8 } & $\mathrm{N}$ & $\%$ & $\mathrm{~N}$ & $\%$ & $\mathrm{~N}$ & $\%$ & P-VALOR \\
\cline { 2 - 8 } & & & & & & & \\
\hline SEXO & & & & & & & 0,492 \\
\hline FEMININO & 22 & 52,4 & 17 & 60,7 & 39 & 55,7 & \\
\hline MASCULINO & 20 & 47,6 & 11 & 39,3 & 31 & 44,3 & \\
\hline & & & & & & & \\
\hline DISFUNÇÃO & & & & & & & 0,233 \\
\hline $\begin{array}{l}\text { SISTÓLICA } \\
\text { (FEVEE<50\%) }\end{array}$ & 20 & 55,6 & 17 & 70,8 & 37 & 61,7 & \\
\hline $\begin{array}{l}\text { DIASTÓLICA } \\
\text { (FEVE> 50\%) }\end{array}$ & 16 & 44,4 & 07 & 29,2 & 23 & 38,9 & \\
\hline & & & & & & & \\
\hline IMC & & & & & & & 0,602 \\
\hline BAIXO PESO & 03 & 7,3 & 02 & 7,1 & 05 & 7,2 & \\
\hline NORMAL & 28 & 68,3 & 15 & 53,6 & 43 & 62,3 & \\
\hline
\end{tabular}

\begin{tabular}{|l|l|l|l|l|l|l|l|}
\hline $\begin{array}{l}\text { ACIMA DO } \\
\text { PESO }\end{array}$ & 04 & 9,8 & 05 & 17,9 & 09 & 13,0 & \\
\hline OBESO & 06 & 14,6 & 06 & 21,4 & 12 & 17,4 & \\
\hline
\end{tabular}

* Classe Funcional (CF) de acordo com New York Heart Association (NYHA)

A média de peso dos pacientes do estudo foi de $65,16 \mathrm{~kg}( \pm 14,31)$ e a altura de $1,60 \mathrm{~cm}( \pm 0,068)$. Enquanto que a idade e a Fração de Ejeção do Ventrículo Esquerdo (FEVE), a média foi de $54( \pm 15)$ anos e $46,74 \%( \pm 19,23)$, respectivamente.

De acordo com a Etiologia da Insuficiência Cardíaca dos pacientes (Tabela 2), a causa principal foi a Miocardiopatia Chagásica (MCC), com 55,9\%, seguido pelas Valvulopatias (24,6\%) e da Hipertensão (HAS), com $14,5 \%$, sendo que onze desses pacientes apresentaram mais de uma etiologia.

Tabela 2 - Etiologia da Insuficiência Cardíaca Congestiva, de acordo com os pacientes do Ambulatório Silva e Lima do HSI, 2010.

\begin{tabular}{cll}
\hline \hline Variáveis & $\mathrm{N}$ & $\%$ \\
\hline \hline Etiologia & & \\
\hline MCC & 38 & 55,9 \\
\hline Valvulopatias & 17 & 24,6 \\
\hline HAS & 10 & 14,5 \\
\hline IAM & 07 & 10,1 \\
\hline MCD & 03 & 4,4 \\
\hline Viral & 01 & 1,4 \\
\hline Periparto & 01 & 1,4 \\
\hline \hline D. Congênita & 01 & 1,4 \\
\hline \hline
\end{tabular}

MCC - Miocardiopatia Chagásica; HAS - Hipertensão; IAM - Infarto Agudo do Miocárdio; MCD - Miocardiopatia Dilatada; D. Congênita Doença Congênita

$\mathrm{Na}$ avaliação dos fatores de risco cardiovasculares apresentados pelos pacientes (Tabela 3 ), houve uma maior incidência de ex-tabagistas (42,9\%), enquanto que nas comorbidades apresentadas pelos pacientes de CF II e CF III observou-se uma maior frequência em pacientes portadores de HAS $(61,4 \%)$, DM $(18,6 \%)$ e Insuficiência Vascular (11,4\%). Os demais, uma menor prevalência. 
Tabela 3 - Relação dos Fatores de Risco e Comorbidades apresentadas pelos pacientes CFII e CFIII do Ambulatório Silva e Lima do HSI, 2010.

\begin{tabular}{ccc}
\hline \hline & $\mathrm{N}$ & $\%$ \\
\hline Variáveis & Fator de Risco & \\
\hline Ex-tabagismo & 30 & 42,9 \\
\hline Dislipidemia & 07 & 10,0 \\
\hline \hline Tabagismo & 04 & 5,7 \\
\hline \hline HAS & Comorbidades \\
\hline \hline DM & 43 & 61,4 \\
\hline \hline Insuficiência & 13 & 18,6 \\
\hline Vascular & 08 & 11,4 \\
\hline \hline AVE & 06 & 8,6 \\
\hline \hline Febre Reumática & 05 & 7,1 \\
\hline \hline Neoplasia & 02 & 2,9 \\
\hline \hline DPOC & 02 & 2,9 \\
\hline \hline IRA & 01 & 1,4 \\
\hline \hline IRC & 01 & 1,4 \\
\hline \hline Reumatismo & 01 & 1,4 \\
\hline \hline
\end{tabular}

HAS - Hipertensão; DM - Diabetes Mellitus; AVE - Acidente Vascular Encefálico; DPOC - Doença Pulmonar Obstrutiva Crônica; IRA - Insuficiência Renal Aguda; IRC - Insuficiência Renal Crônica.

$\mathrm{Na}$ classificação de acordo com as variáveis clínicas do desempenho físico após o Teste de Caminhada de 6 minutos dos pacientes analisados (Tabela 4), observou-se que não houve diferença nos valores da Pressão Arterial Sistólica e Diastólica e Frequência Cardíaca se mantendo estável durante toda a realização do teste, bem como na distância percorrida de ambas as classes. Entretanto, o T-Student Test mostrou que houve diferença estatística na Escala de Borg $(p=0,005)$.

No resultado da distância percorrida, o Meann - Whitney Test mostrou que não houve significância $(p=0,144)$ em relação às classes funcionais, sendo que a mediana no primeiro intervalo interquartil foi de 50 metros e no terceiro intervalo, 130 metros.

$\mathrm{Na}$ Qualidade de Vida de acordo com a CF II e CF III dos pacientes analisados (Tabela 5), mostra que no grupo de CF II a média da capacidade física foi de $20,92( \pm 10,25)$ e no grupo de CF III foi de 28,60 ( \pm $7,95)$, mostrando que houve diferença estatisticamente significante nos resultados estudados $(p=0,001)$.

Em relação à capacidade psicológica e geral, no grupo de CF II a média foi de 12,09 $( \pm 7,26)$ e 16,21 ( \pm $7,28)$ e na CF III, a média foi de $14,53( \pm 7,12)$ e 19,00
$( \pm 7,06)$, respectivamente. Entretanto, essa diferença não foi estatisticamente significante para capacidade psicológica $(p=0,170)$ e geral $(p=0,117)$.

Tabela 4 - Categorização das Classes Funcionais de acordo com os parâmetros clínicos no Teste de Caminhada de 6 minutos dos pacientes do Ambulatório Silva e Lima do HSI, 2010.

\begin{tabular}{|l|l|l|l|}
\hline \multirow{2}{*}{$\begin{array}{c}\text { Parâmetros } \\
\text { Clínicos }\end{array}$} & \multicolumn{3}{|c|}{ Classe Funcional (NYHA) } \\
\cline { 2 - 4 } & & \multicolumn{1}{|c|}{ III $(\mathrm{n}=28)$} & \\
\cline { 2 - 4 } & Média & Média & p- valor \\
\hline PAS I * & $120,71 \pm 22,67$ & $116,36 \pm 24,12$ & 0,445 \\
\hline PAS II ** & $124,76 \pm 24,81$ & $124,76 \pm 24,81$ & 0,900 \\
\hline PAD I*** & $77,62 \pm 13,21$ & $77,50 \pm 13,11$ & 0,971 \\
\hline PAD II $\dagger$ & $80,00 \pm 11,47$ & $82,69 \pm 16,13$ & 0,425 \\
\hline FC I †† & $68,54 \pm 15,38$ & $70,36 \pm 12,97$ & 0,609 \\
\hline FC II ††† & $79,05 \pm 15,88$ & $78,11 \pm 20,52$ & 0,830 \\
\hline SATO2 I $\times$ & $97,49 \pm 1,70$ & $97,93 \pm 1,18$ & 0,240 \\
\hline SATO2 II $\times \times$ & $97,57 \pm 2,19$ & $96,96 \pm 3,21$ & 0,351 \\
\hline FCM $\times \times \times$ & $166,57 \pm 13,78$ & $165,39 \pm 17,05$ & 0,751 \\
\hline DISTÂNCIA $\infty$ & $104 \pm 52$ & $92 \pm 55$ & 0,428 \\
\hline $\begin{array}{l}\text { ESCALA DE } \\
\text { BORG }\end{array}$ & $10,60 \pm 3,02$ & $12,58 \pm 2,43$ & 0,005 \\
\hline
\end{tabular}

* Pressão Arterial Sistólica em repouso antes do T6'; ** Pressão Arterial Sistólica depois do T6'; ${ }^{* \star *}$ Pressão Arterial Diastólica em repouso antes do T6'; † Pressão Arterial Diastólica depois do T6'; ††Frequência Cardíaca em repouso antes do T6'; †††Frequência Cardíaca depois do T6'; $\times$ Saturação de Oxigênio em repouso antes do T6'; ×xSaturação de Oxigênio depois do T6'; ×xx Frequência Cardíaca Máxima; $\infty$ Distância Percorrida em metros.

Tabela 5 - Qualidade de Vida de acordo com a CF II e CF III de pacientes do Ambulatório Silva e Lima do HSI, 2010.

\begin{tabular}{|l|l|l|l|}
\hline \multirow{3}{*}{$\begin{array}{l}\text { Qualidade de } \\
\text { Vida }\end{array}$} & \multicolumn{1}{|c|}{ II $(\mathrm{n}=42)$} & \multicolumn{1}{|c|}{ III $(\mathrm{n}=28)$} & \\
\cline { 2 - 4 } & & & \\
\cline { 2 - 4 } & Média & Média & $\mathrm{p}$ - valor \\
\hline Escore Global & $49,26 \pm 21,24$ & $62,14 \pm 17,83$ & 0,010 \\
\hline $\begin{array}{l}\text { Capacidade } \\
\text { Física }\end{array}$ & $20,92 \pm 10,25$ & $28,60 \pm 7,95$ & 0,001 \\
\hline $\begin{array}{l}\text { Capacidade } \\
\text { Psicológica }\end{array}$ & $12,09 \pm 7,26$ & $14,53 \pm 7,12$ & 0,170 \\
\hline $\begin{array}{l}\text { Capacidade } \\
\text { Geral }\end{array}$ & $16,21 \pm 7,28$ & $19,00 \pm 7,06$ & 0,117 \\
\hline
\end{tabular}

\section{DISCUSSÃO}

Segundo o Ministério da Saúde em 2010, cerca de três milhões de pessoas no Brasil estão infectadas com a doença de Chagas, sendo que a Bahia é a re- 
gião de risco para transmissão vetorial da doença, que leva às complicações cardíacas. Este dado corrobora com o presente estudo, no qual a Miocardiopatia Chagásica é de $55,9 \%$, apresentando alta incidência em relação à etiologia da Insuficiência Cardíaca.

Quanto à prevalência da disfunção sistólica foi predominante na classe III. Conforme o estudo de Schmidt (2001), a presença da HAS favorece a hipertrofia ventricular, reduzindo as dimensões contráteis, prejudicando a complacência. Desta forma, em casos avançados da doença, a disfunção diastólica precede a disfunção sistólica, que é uma manifestação clínica da IC.

Quando comparada à QDV entre a CF II e a CF III, o resultado mostrou diferenças significativas na capacidade física, onde na CF II o menor valor confirma a mínima interferência da dispneia para realizar as atividades habituais. Bem como foi observado no TC6' na CFII, apresentando a maior distância percorrida e o menor esforço percebido. Conforme Camargo e cols (2009), à distância percorrida no Teste de Caminhada de 6 minutos, mostraram uma correlação significativa com a classe funcional e gravidade da doença. Com isso, o resultado do estudo indicou que não houve diferença nas variáveis clínicas, se mantendo estáveis em ambas as Classes Funcionais.

Corroborando com Sakir Arslan et al, 2007, que há influência do prognóstico de mortalidade quanto à distância percorrida em ralação com a fração de ejeção menor que $30 \%$. No presente estudo, quando comparado o desempenho físico através do teste de caminhada, quatro pacientes não concluíram o percurso representado pela Classe III, no qual três destes apresentavam disfunção sistólica com FEVE $<30 \%$, indo a óbito após seis meses da entrevista.

Entre as classes funcionais (NYHA), a distância atingida no TC6' está de acordo com dados descritos por Sousa (2008), que atribui a fraca associação entre classe funcional e distância caminhada à imprecisão da escala da NYHA.

\section{CONCLUSÃO}

A IC tem alta prevalência nas doenças cardíacas, sendo que a incidência mais frequente é a miocardiopatia chagásica. Contudo, este estudo mostra que a baixa qualidade de vida está diretamente relacionada à presença da alteração da estrutura funcional do coração nos indivíduos analisados, provocando limitações físicas e interpessoais, principalmente em pacientes portadores da CF III.

O conhecimento da evolução dos estágios da IC possibilita um melhor tratamento e maior atenção, especialmente aos grupos de maior risco, permitindo controlar a evolução da doença. Com isso, reduz a sua morbimortalidade, por meio de tratamento clínico ou cirúrgico, através da melhora dos sinais clínicos, associados às limitações cotidianas e à qualidade de vida dos pacientes.

Todavia, este estudo consentiu obter informações das dificuldades apresentadas por esta amostra de pacientes, bem como identificar a qualidade de vida e as limitações físicas diante da gravidade da insuficiência cardíaca.

Vale ressaltar que este estudo servirá como base de conhecimento para novas pesquisas, a fim de acrescentar variáveis clínicas no prognóstico da IC.

\section{REFERÊNCIAS}

ATHANASIOS, T., et al. Effects of interval exercise training on respiratory drive in patients with chronic heart failure. Respiratory medicine v. 104. n. 10. p. 155765,2010.

BANSAL, V. et al. Modifying Track Layout From Straight to Circular has a Modest Effect on the 6-min Walk Distance. v. 133. p. 1155-1160,2008.

BERISHA, V. e cols. Ecocardiografia e Teste de Caminhada de 6 Minutos na Disfunção Sistólica do Ventrículo Esquerdo. Arquivo Brasileiro Cardiologia 2009;92(2):127-134.

BOCCHI, E.A. et al. I Diretriz Latino-Americana para Avaliação e Conduta na Insuficiência Cardiáca Descompensada. Arquivo Brasileiro Cardiologia. 2005;85.

CAMARGO e cols. Validation of a treadmill six-minute walk test protocol for the evaluation of patients with pulmonary arterial hypertension. Jornal Brasileiro Pneumologia:35(5):423430,Maio2009.

CARRARA, D. Avaliação prospectiva da qualidade de vida em pacientes com miocardiopatia dilatada submetidos à ventriculectomia parcial esquerda [Dissertação]. São Paulo: Universidade de São Paulo; 2001.

CARVALHO, V.O. e cols. Validação da Versão em Português do Minnesota Living with Heart Failure Questionnaire. Arquivo Brasileiro Cardiologia 2009;93 (1):39-44.

CHIAPPA, G.R. et al. Inspiratory Muscle Training Improves Blood Flow to Resting and Exercising Limbs in Patients With Chronic Heart Failure. Journal of the American College of Cardiology. v. 51, n. 17. p. 1663-71, 2008.

CRAPO, R.O. e cols. ATS statement: Guidelines for the six-minute walk test. American Journal of Respiratory and Critical care Medicine, 2002; 166: 111-117.

DANTAS, R.A.S. e cols. Research on quality of life: review on the scientific production of public universities in São Paulo State Revista Latino-Americana Enferma- 
gem, vol.11, n.4, Ribeirão Preto, July/Aug. 2003

ENRIGHT, P.L.The six-minute walk test. Respiratory Care 2003; 48:783-785.

FERRANS, C.E; POWERS M.J. Psychometric assessment of the quality of life index. Res Nurs Health. 1992; 15: 29-38.

FIGUEROA, M.S. and PETERS, J.I. Congestive Heart Failure: Diagnosis, Pathophysiology, Therapy, and Implications for Respiratory Care RespiratoryCare,April,2006.vol.51, $\mathrm{n}^{\circ} 4<$

FUMAGALLI, E. e cols. Utilização do teste de caminhada de 6 minutos no manejo da hipertensão pulmonar. Arquivo Brasileiro Cardiologia, vol. 95 n.1. São Paulo, July 2010.

GUYTON, A.C. e HALL, J.E. Tratado de Fisiologia Médica, Ed. Guanabara Koogan, 2008, $9^{a}$ edição: 245253, 286.

KUZNETSOVA, T. e cols, Prevalence of Left Ventricular Diastolic Dysfunction in a General Population Circulation: Heart Failure. 2009; 2:105-112 Fevereiro/2009.

LI, A.M. e cols. The six - minute walk test healthy children: reliability and validity. Eu Respiratory Journal. 2005; 25: 1057 - 1060.

LOPES, V.P. et al. Caracterização da atividade física habitual em adolescentes de ambos os sexos através de acelerometria e pedometria. Revista Paulista Educação Física, São Paulo, 17(1): 51-63,jan./jun2003

MILLER, K. e cols. Quality of life in bridge-to-transplant patients with chronic heart failure after implantation of an axial flow ventricular assist device. Congest Heart Fail. 2004; 10 (5): 226-9.

NOGUEIRA, I.D.B e cols. Correlação entre qualidade de vida e capacidade funcional na insuficiência cardíaca. Arquivo Brasileiro Cardiologia. vol. 95 n. 2, São Paulo. Aug 2010.

OLIVEIRA, M. et al. Avaliação da Atividade Física em contextos epidemiológicos. Uma revisão da validade e fiabilidade do acelerômetro Tritac - R3D, do pedômetro Yamax Digi Walk e do Questionário de Baecke. Revista Portuguesa de Ciência do Desporto, vol.1. (3). 73-78, 2003.

PILON, A.F. Qualidade de vida e formas de relacionamento homem/mundo. Revista Brasileira Saúde Esc. 1992; 2 (3): 117-25.

PINTO, C.N.C. Níveis de atividade física e prevalência de excesso de peso e obesidade em crianças e jovens do Conselho de Matosinhos. Porto, 2008.

RIEGEL, B. et al. The Minnesota living with heart failure questionnaire: sensitivity to differences and responsiveness to intervention intensity in a clinical population. Nurs Res. 2002; 51 (4): 209-18.

SACCOMANN, I.C; CINTRA, F.A; GALLANI, M.C.
Psychometric properties of the Minnesota Living with Heart Failure--Brazilian version--in the elderly. Quality Life Res. 2007; 16 (6): 997-1005.

SANTOS, J.J.A. e cols, Quality of Life and Clinical Indicators in Heart Failure: a Multivariate Analysis, Arquivo Brasileiro Cardiologia 2009; 93(2) : 159-166

SCHMIDT, A. Systolic and diastolic heart failure in hypertensive patients differential diagnosis and treatment Revista Brasileira Hipertensão vol 8(4): Outubro/ Dezembro 2001.

SEIDL, E.M.F. e ZANNON, C.M.L.C. Qualidade de vida e saúde: aspectos conceituais e metodológicos. Caderno Saúde Pública, vol. 20, n. 2. Rio de Janeiro Mar./Apr. 2004.

SMITH, S.C. e cols AHA/ACC Scientific Statement: AHA/ACC guidelines for preventing heart attack and death in patients with atherosclerotic cardiovascular disease: 2001 update: a statement for healthcare professionals from the American Heart Association and the American College of Cardiology. Circulation.2001;104:15771579.

SOARES, D.A. et al. Qualidade de vida de portadores de insuficiência cardíaca. Acta Paulista de Enfermagem 2008; 21(2): 2438.

SOUSA, L.A.P. Associação entre fatores fisiopatológicos, Capacidade e desempenho funcionais na Doença de Chagas. Tese apresentada ao Programa de Pós-graduação em Infectologia e Medicina Tropical da Faculdade de Medicina da Universidade Federal de Minas Gerais para obtenção de título de Doutor. Belo Horizonte, 2008.

SWARTZ, A.M e cols. Validity of Physical Activity Monitors in Assessing Energy Expenditure in Normal, Overweight, and Obese Adults. International Open Sports Sci Journal. 2009; 2:58-64.

SWARTZ, A.M; BASSETT, D.R.Jr; MOORE, J.B; THOMPSON, D.L; STRATH, S.J. Effects of body mass index on the accuracy of an electronic pedometer. International Journal Sports Medicine 2003;24:588-592. [PubMed: 14598195]

WILLIAM, E. D.T.; SCOTT, L.B. Physical Therapists as Providers of Care: Exercise Prescriptions and Resultant Outcomes in Cardiac and Pulmonary Rehabilitation Programs in New York State. Cardiopulmonary Physical Therapy Journal. Vol 19, No 2, June 2008.

1- Serviço de Fisioterapia do HSI Endereço para correspondência: fisiosandraoliveira@hotmail.com 


\section{Eventos Fixos}

\begin{tabular}{|c|c|c|c|c|}
\hline Sessão & Local & Dias da Semana & Responsável & Horário \\
\hline $\begin{array}{l}\text { Sessão de Hemodinâmica e } \\
\text { Cardiologia Intervencionista }\end{array}$ & Auditório & Todas as $2^{\text {as }}$ & Prof. Dr. José Carlos Brito & 07:30 às 09:00 \\
\hline Sessão de Casos Clínicos & Auditório & Todas as $3^{\text {as }}$ & Prof. Dr. Gilson Feitosa & $10: 00$ às $13: 00$ \\
\hline Sessão de Cardio/Pneumo & Auditório & $3^{\mathrm{a}}$ ou $5^{\mathrm{a}}$ & $\begin{array}{l}\text { Prof. Dr. Gilson Filho / Prof. } \\
\text { Dr. Marcel Lima }\end{array}$ & $12: 00$ às $13: 00$ \\
\hline Sessão de Atualização & Auditório & Todas as $5^{\text {as }}$ & Prof. Dr. Gilson Feitosa & $11: 00$ às $13: 00$ \\
\hline Sessão de Clínica Médica & ST1 & Todas as $3^{\text {as }}$ & Prof. Dr. Mateus Rosário & $13: 30$ às $15: 30$ \\
\hline Sessão de Anestesia & ST1 & Todos os dias & Prof. Dr. Jedson & 07:00 às $08: 30$ \\
\hline Sessão de Medicina Intensiva & ST4 & Todas as $4^{\text {as }}$ & Prof. Dr. Edson Marques & $11: 00$ às $13: 00$ \\
\hline Sessão de Arritmologia & ST3 & Todas as $2^{\text {as }}$ & Prof. Dr. Bráulio Pinna & $13: 00$ às $15: 00$ \\
\hline Sessão de Arritmologia & ST4 & Todas as $5^{\text {as }}$ & Prof. Dr. Alessandre Rabelo & $13: 00$ às $15: 00$ \\
\hline Sessão de Clínica Médica / Endócrino & ST4 & Todas as $5^{\text {as }}$ & Profa. Dra. Alina Feitosa & 09:30 às $11: 00$ \\
\hline Curso de Semiologia & ST2 & Todas as $2^{\text {as }}$ e $4^{\text {as }}$ & Prof. Dr. Augusto Almeida & $14: 00$ às $15: 30$ \\
\hline $\begin{array}{l}\text { Sessão de Gastroenterologia } \\
\text { Digestiva }\end{array}$ & ST2 & Todas as $3^{\text {as }}$ & Prof. Dr. Jayme Fagundes & $19: 00$ às $20: 00$ \\
\hline Sessão de Pneumologia & SENEP & $\begin{array}{l}\text { Todas as } 5^{\text {as }} \text { (Última } 5^{a} \text { feira } \\
\text { - Anatomoclínica - Prof. Dr. } \\
\text { Jamocyr Marinho) }\end{array}$ & Prof. Dr. Guilhardo Ribeiro & 08:00 às $10: 00$ \\
\hline Sessão de Pneumologia & ST2 & Todas as $6^{\text {as }}$ & Prof. Dr. Jamocyr Marinho & $10: 30$ às $12: 00$ \\
\hline Sessão de Cardiologia Pediátrica & ST2/ST4 & Todas as $5^{\text {as }}$ e $6^{\text {as }}$ & Profa. Dra. Anabel & 07:00 às 09:00 \\
\hline Serviço de Coloproctologia & ST1 & Todas as $3^{\text {as }}$ & Dr. Ramon Mendes & $19: 00$ às $21: 00$ \\
\hline Serviço de Imagem & ST2 & Todas as $5^{\text {as }}$ & $\begin{array}{l}\text { Profa. Dra. Cristiane } \\
\text { Abbenhoussen }\end{array}$ & $12: 00$ às $14: 00$ \\
\hline Serviço de Ortopedia & ST3 & Todas as $4^{\text {as }}$ & Prof. Dr. Rogério & $19: 00$ às $21: 00$ \\
\hline Serviço de Ortopedia & ST3 & Todas as $5^{\text {as }}$ & Prof. Dr. Rogério & 06:30 às 08:00 \\
\hline Sessão de Cirurgia Geral & SENEP & Todas as $3^{\text {as }}$ & Prof. Dr. André Ney & $08: 00$ às $10: 00$ \\
\hline Sessão de Neurologia & ST4/SENEP & Todas as $5^{\text {as }}$ e $6^{\text {as }}$ & Prof. Dr. Pedro Antônio & $16: 00$ às $18: 00$ \\
\hline Sessão de Otorrinolaringologia & ST4 & Todas as $3^{\text {as }}$ e $6^{\text {as }}$ & Prof. Dr. Nilvano & 07:00 às 09:00 \\
\hline Sessão de Urologia & SENEP & Todas as $3^{\text {as }}, 4^{\text {as }}$ e $6^{\text {as }}$ & Prof. Dr. Eduardo Café & 07:00 às 09:00 \\
\hline
\end{tabular}




\begin{tabular}{|c|c|c|c|}
\hline Classificação manuscritos & $\begin{array}{l}N^{\circ} \text { máximo } \\
\text { de autores }\end{array}$ & $\begin{array}{l}\text { TÍTULO: } N^{\circ} \text { máximo de } \\
\text { caracteres com espaço }\end{array}$ & $\begin{array}{l}\text { RESUMO: } N^{\circ} \text { máximo } \\
\text { de palavras }\end{array}$ \\
\hline Editorial & 2 & 100 & 0 \\
\hline Atualização de tema & 4 & 100 & 250 \\
\hline Resumo de artigos publicados pelo HSI & 10 & 100 & 250 \\
\hline Relato de casos & 6 & 80 & 0 \\
\hline
\end{tabular}

Continuação:

\begin{tabular}{|c|c|c|c|}
\hline Classificação manuscritos & $\begin{array}{l}N^{\circ} \text { máximo de palavras } \\
\text { com referência }\end{array}$ & $\begin{array}{l}\mathrm{N}^{\circ} \text { máximo de } \\
\text { referências }\end{array}$ & $\begin{array}{l}\mathrm{N}^{\circ} \text { máximo de } \\
\text { tabelas ou figuras }\end{array}$ \\
\hline Editorial & 1.000 & 10 & 2 \\
\hline Atualização de tema & 6.500 & 80 & 8 \\
\hline Resumo de artigos publicados pelo HSI & 1.500 & 10 & 2 \\
\hline Relato de casos & 1.500 & 10 & 2 \\
\hline
\end{tabular}




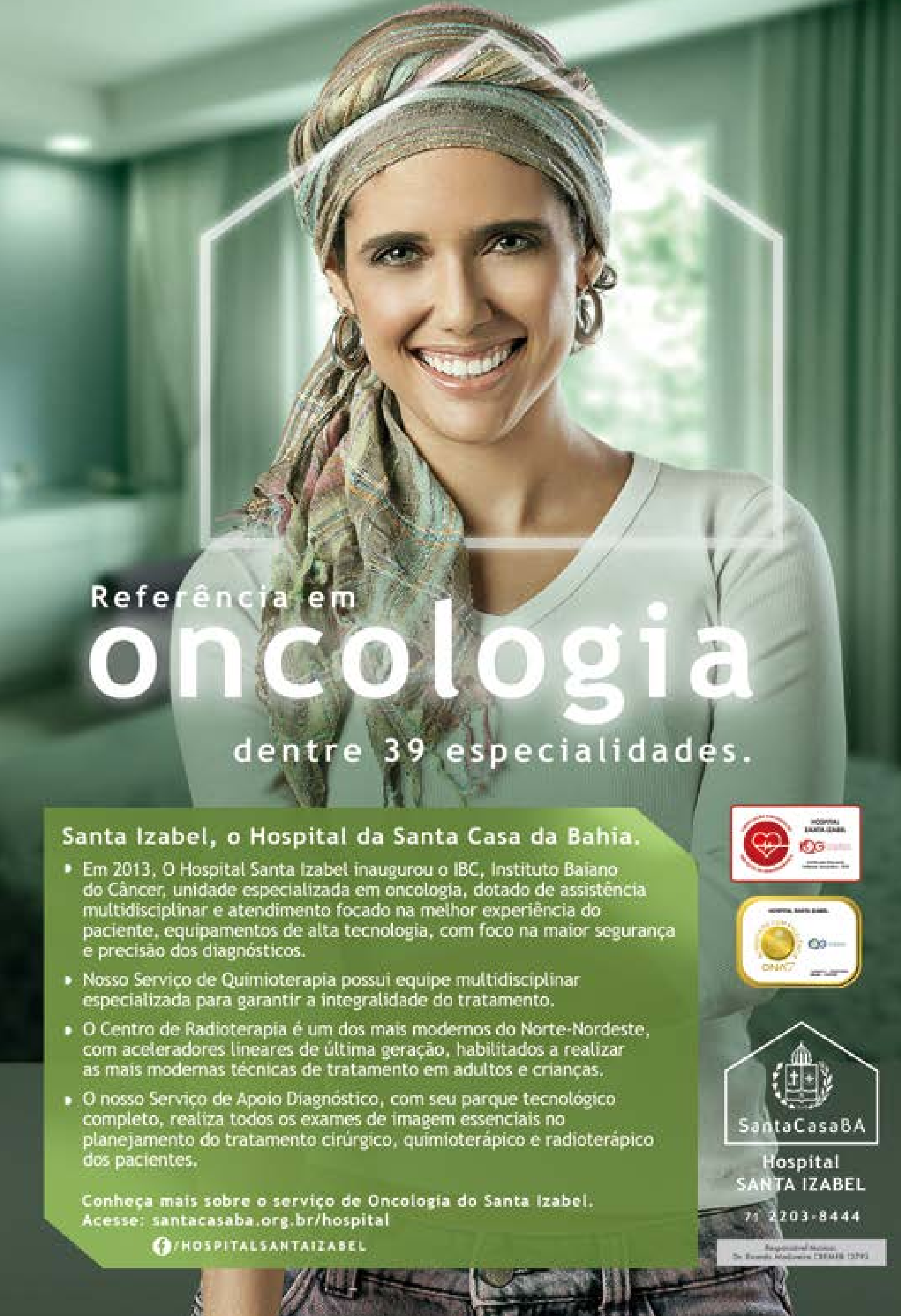




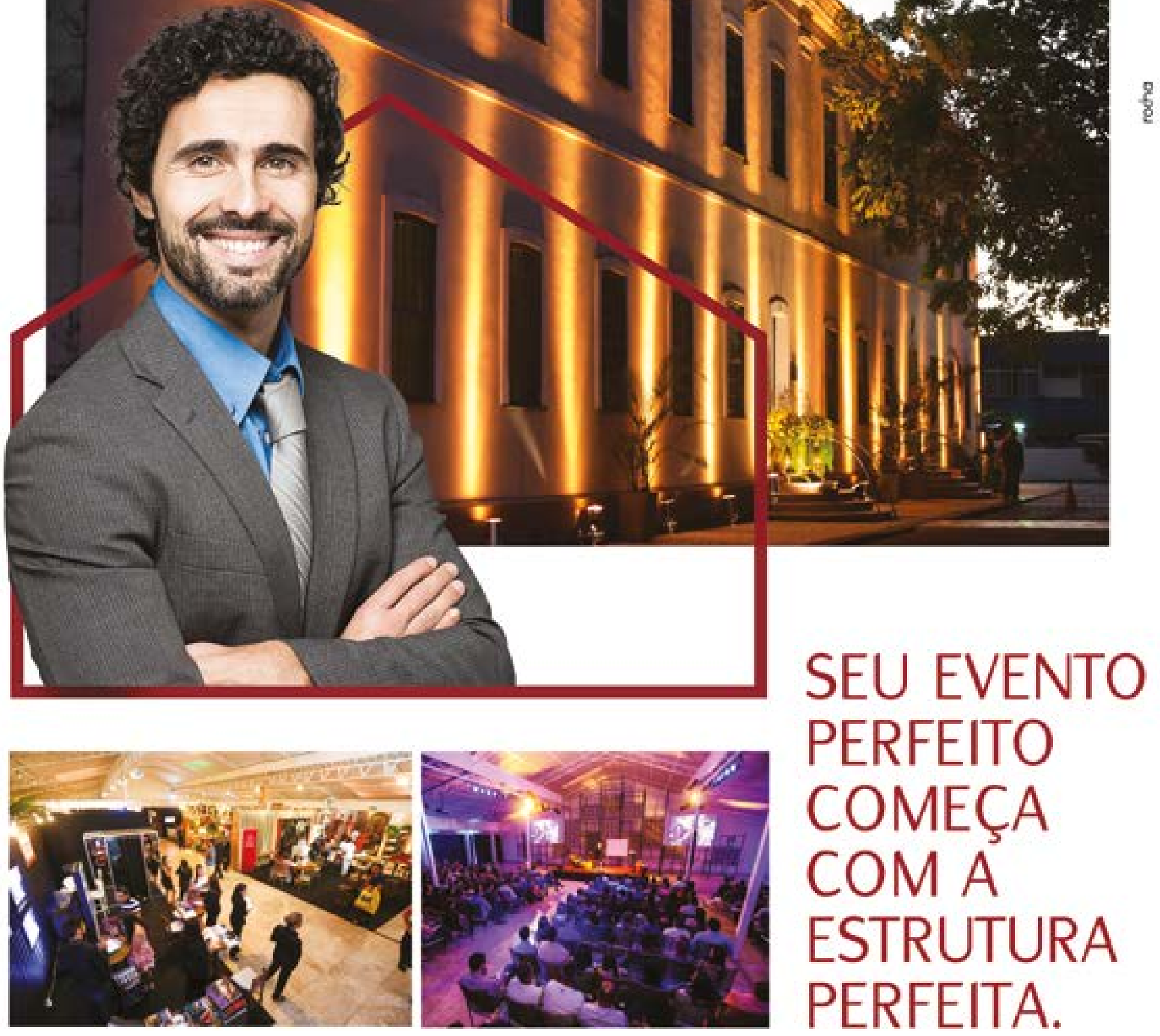

Salào principal com capacidade para 1.500 pessoas sentadas

3 salöes climatizados.

somando $325 \mathrm{~m}$ ?

Capela N. Sra. das Vitórias

idesde 1876) climatizada

Area verde com $660 \mathrm{~m}$
Estacionamento com mais

de 250 vagas internas e área VIP para 12 veiculos

Brinquedoteca

Camarim e sala de apoio

Cozinha industrial equipada

\section{Charme, sofisticaçăo e a} melhor estrutura. O Cerimonial Rainha Leonor, na Pupileira. é o mais completo da cidade. Sua versatilidade em espaços adaptáveis permite acomodar, com - maior conforto, desde aniversários e festas de formatura até eventos corporativos, como feiras e convençð̄es. $\mathrm{O}$ ambiente ideal para o seu evento. Venha conhecer.

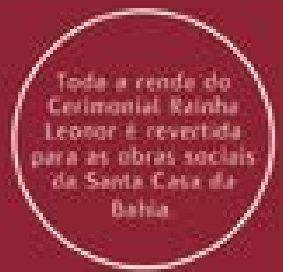

Av. Joana Angelica, 79, Nazare (Pupileira), Sakrodor-8A CEP, 40.050-001. Tel: (71) $22039663 /(71) 22039647$

F/CerinonialReintiaLeonot 0 eRaintoleonoicerimonial

www.santacasabarorgibr/cerimonial
Cerimonial

RAINHA LEONOR

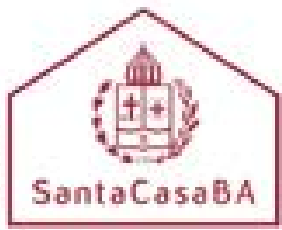

